\title{
SISPRENATAL COMO FERRAMENTA FACILITADORA DA ASSISTÊNCIA À GESTANTE: REVISÃO INTEGRATIVA DA LITERATURA
}

\author{
SISPRENATAL AS A FACILITATOR TOOL OF PREGNANCY ASSISTENCE: \\ AN INTEGRATIVE REVIEW OF THE LITERATURE
}

\section{Julia Tavares de Carvalho Barbosa ${ }^{a^{*}}$, Thalita Neiva Breda Vettori ${ }^{\mathrm{b}^{*}}$, Bruna Lopes Saldanha ${ }^{\mathrm{c}^{*}}$, Raí Moreira Rocha ${ }^{\mathrm{d}^{*}}$, André Luiz de Souza Braga ${ }^{\mathrm{e}^{*}}$, Marilda Andrade ${ }^{\mathrm{f}^{*}}$}

ajuliatcb@ig.com.br, bthalibreda@gmail.com, cbrunasaldanha@gmail.com, ${ }^{d}$ moreirarocha958@gmail.com, eandre.braga@globo.com, 'marildaandrade@uol.com.br

"Escola de Enfermagem Aurora de Afonso Costa, Universidade Federal Fluminense - Niterói (RJ), Brasil

Data de entrada do artigo: 06/10/2013

Data de aceite do artigo: 19/03/2014

\section{RESUMO}

Introdução: O SISPRENATAL é um instrumento de avaliação e permite o monitoramento da assistência pré-natal e puerperal fornecidas pelos serviços de saúde à mulher e ao recém-nascido "desde o primeiro

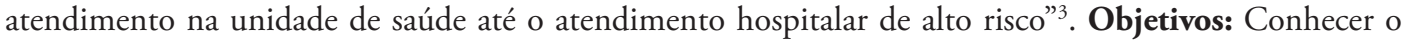
funcionamento do SISPRENATAL na atenção à mulher grávida, pois ao descrever como esse instrumento facilita a assistência à saúde da gestante por meio das informaçôes geradas. Materiais e métodos: Tratase de uma pesquisa exploratória, com abordagem qualitativa, cujo método empregado para a coleta de dados foi a revisão integrativa com busca das obras potenciais nas bases científicas de dados. Resultados: A assistência pré-natal tem como finalidade a redução da morbidade e mortalidade materna e infantil, pela implementação de medidas e realizaçáo de procedimentos mínimos definidos pelo PHPN. O SISPRENATAL é o instrumento que foi criado para a obtenção de informaçóes acerca da saúde durante o parto pré-natal e puerpério, permitindo, por monitoração do cumprimento dos procedimentos mínimos preconizados pelo PHPN, que haja a "implementação de repasse de verbas para os municípios a cada gestante que cumpra esses requisitos mínimos" ${ }^{12}$. Conclusão: Por meio da revisão integrativa realizada neste estudo, pode-se constatar a significativa quantidade de publicaçôes encontradas nas bases científicas de dados que abordam o tema cuidado de gestante e SISPRENATAL. O sistema possibilita, por meio das informaçôes geradas, o planejamento, a (re)organização dos serviços de saúde e a manutenção da assistência de qualidade à gestante.

Palavras-chave: Sistema de gerenciamento de dados; administração dos serviços de saúde; enfermagem; gestantes.

\begin{abstract}
Introduction: SISPRENATAL is an assessment tool that allows monitoring of prenatal and postpartum health services provided by the services of women and newborn care since the first doctor's appointment at the health unit to the high-risk hospital care. Objectives: To understand the operation of the SISPRENATAL in the assistance of pregnant women by the description of how this tool facilitates healthcare for pregnant women through the information generated. Materials and Methods: This was an exploratory, qualitative approach, whose method used to collect data was the integrative review, searching for potential works on scientific data. Results: Prenatal care aims to reduce morbidity and maternal and child mortality through the implementation of measures and minimal performing procedures defined by PHPN. SISPRENATAL is a tool that was created to obtain information about health during prenatal, labor and postpartum, enabling monitoring the compliance with the minimum procedures recommended by PHPN, the
\end{abstract}


implementation of transfer of funds to municipalities to every pregnant woman who meets these minimum requirements. Conclusion: Through integrative review conducted in this study, it can be seen a significant amount of publications found in scientific bases that address the care of pregnant women and SISPRENATAL. The system allows the planning, (re)organization of health services and maintenance of the quality care to pregnant women through the information that are generated.

Keywords: System management data; management of health services; nursing; pregnant women.

\section{Introdução}

A informação constitui uma ferramenta essencial para ações dentro do sistema de saúde. Por ela, torna-se possível o desenvolvimento do processo informação, decisão e ação. A informação é gerada a partir dos dados que são a matéria-prima da produção de informação. Por meio de um quantitativo referente a um fato ou circunstância, é possível suscitar o conhecimento a partir da interpretação dos dados ${ }^{1}$. Os Sistemas de Informação em Saúde (SIS), por sua vez, são o suporte para a organização das ações do serviço de saúde, pois subsidiam a função administrativa das unidades de saúde e, consequentemente, corrobora para a melhoria da assistência ao indivíduo e populaçáo ${ }^{2}$.

Para o gerenciamento das açóes voltadas às gestantes e assistência a esse grupo populacional, foi desenvolvido, pelo DATASUS, um sistema que tem como finalidade permitir o acompanhamento oportuno das gestantes inseridas no Programa de Humanização no Pré-Natal e Nascimento (PHPN) do Sistema Único de Saúde (SUS), o SISPRENATAL. Neste sistema, estão definidos os procedimentos mínimos necessários para uma adequada assistência pré-natal, permitindo o acompanhamento da gestante do início da gestação até a consulta de puerpério ${ }^{3}$. O SISPRENATAL é um instrumento de avaliação e permite o monitoramento da assistência pré-natal e puerperal fornecido pelos serviços de saúde à mulher e ao recém-nascido "desde o primeiro atendimento na unidade de unidade de saúde até o atendimento hospitalar de alto risco"3.

O Programa de Humanização do Pré-Natal e Nascimento (PHPN) foi criado pelo Ministério da Saúde em 2000 e tem como objetivos assegurar o acesso universal à atenção de qualidade a gestação, parto, puerpério e período neonatal; reduzir taxas de morbimortalidade materna e infantil; e complementar medidas já adotadas para aprimorar a assistência à gestante, na perspectiva dos direitos básicos de cidadania. $\mathrm{O}$ SISPRENATAL tem por objetivo desenvolver ações de promoção, prevenção de agravos e assistência à saúde de gestantes e recém-natos, ampliando esforços que visem a redução de altas taxas de morbidade e mortalidade materna, perinatal e neonatal, melhorando acesso, cobertura e qualidade do acompanhamento pré-natal ao parto e puerpério e da assistência neonatal, subsidiando municípios, estados e Ministério da Saúde com informaçóes fundamentais para o planejamento, acompanhamento e avaliação das açóes desenvolvidas pelo $\mathrm{PHPN}^{4}$.

A partir desta contextualização e da prática assistencial à mulher gestante na rede básica de saúde do município de Niterói (RJ), emergiram os objetivos da pesquisa que consistem em: conhecer o funcionamento do SISPRENATAL na atenção à mulher grávida; descrever como esse instrumento facilita a assistência à saúde da gestante por meio das informaçóes geradas.

\section{Materiais e métodos}

Trata-se de uma pesquisa exploratória, que tem como desígnio desenvolver, esclarecer e modificar conceitos, visando à elaboração de problemas mais precisos ou formulação de hipóteses para estudos futuros. Este tipo de pesquisa é desenvolvido a fim de proporcionar uma visão geral, aproximando o pesquisador de determinado fenômeno.

A abordagem de escolha para a construção da pesquisa foi a qualitativa, pois se deseja estudar um fato imensurável, ou seja, como incide a utilização do SISPRENATAL e de que forma esse instrumento contribui para a assistência à gestante.

[...] se aplica ao estudo da história, das relaçóes das interpretaçôes, das crenças, das percepçôes e das opiniōes, produtos das interpretaçóes que os humanos fazem a respeito de como vivem, constroem seus artefatos e a si mesmos, sentem e pensam. É caracterizada pela empiria e pela sistematização progressiva do conhecimento até a compreensão da lógica interna do grupo ou do processo em estudo. Por isso também é utilizada para a elaboração de novas hipóteses, construção de indicadores qualitativos, variáveis e tipologias.

O método empregado, para busca e análise das obras que seriam utilizadas no estudo, foi a revisão integrativa, que apresenta como objetivo reunir e sintetizar os resultados da pesquisa sobre determinado tema, de forma ordenada e sistemática, possibilitando que o pesquisador 
aprofunde seus conhecimentos acerca do assunto investigado $^{6}$. Este método apresenta seis etapas, a saber:

$1^{\text {a: }}$ identificação do tema e seleção da questão de pesquisa para a elaboraçáo da revisão integrativa; $2^{a}$ : estabelecimento de critérios para inclusão e exclusão de estudos; $3^{\text {a: }}$ definição das informaçōes a serem extraídas dos estudos selecionados; $4^{\mathrm{a}}$ : avaliação dos estudos incluídos na revisão integrativa; $5^{a}$ : interpretação dos resultados; $6^{a}$ : apresentação da revisão ${ }^{6}$.

A partir destas fases, torna-se possível a busca sistematizada das obras disponíveis nas bases literárias, organização e síntese das obras publicadas, o que permite maior aproximação e apropriação do autor quanto ao tema.

$\mathrm{Na}$ construção do presente artigo, foi realizada a busca bibliográfica por revisão integrativa na Biblioteca Virtual de Saúde (BVS), nas bases de dados: Base de Dados em Enfermagem (BDENF), Literatura LatinoAmericana em Ciências da Saúde (LILACS) e na Scientific Electronic Library Online (SciELO), para a obtenção de obras científicas que apresentassem como abordagem assuntos relacionados a assistência à saúde da gestante, pré-natal e SISPRENATAL.

A partir da definiçáo dos critérios de inclusão - publicações que abordassem o pré-natal, disponíveis na íntegra, nos idiomas português e espanhol, e que tenham sido disponibilizadas dentro do corte temporal de 2008 a 2013 - e os critérios de exclusão da pesquisa - artigos publicados antes do ano de 2008, que estivessem em outro idioma que não o português e o espanhol e não estar disponível na íntegra on-line, sendo realizada a busca pelos seguintes descritores: pré-natal; sistemas de informação, assistência à saúde; humanização da assistência. O quadro 1 mostra o quantitativo de obras encontradas por descritor nas bases de dados.

Quadro 1: Quantitativo de publicações encontradas por base de dados

\begin{tabular}{lccc}
\hline \multicolumn{1}{c}{ Descritores } & \multicolumn{3}{c}{ Bases de Dados } \\
\hline & LILACS & BDENF & SCIELO \\
\hline $\begin{array}{l}\text { Pré-natal } \\
\text { Sistemas de }\end{array}$ & 1.770 & 300 & 1.381 \\
$\begin{array}{l}\text { informação } \\
\text { Assistência à saúde }\end{array}$ & 1.942 & 62 & 688 \\
$\begin{array}{l}\text { Humanização da } \\
\text { assistência }\end{array}$ & 3.586 & 285 & 2.544 \\
TOTAL & 542 & 288 & 152 \\
\hline
\end{tabular}

Posterior à busca das publicações, utilizando os descritores, realizou-se a associação dos descritores a fim de refinar a pesquisa, da seguinte forma: "sistemas de informaçáo" AND "pré-natal"; "pré-natal” AND "assistência à saúde"; "pré-natal" AND "humanização da assistência"; "sistemas de informação" AND "assistência à saúde". Estão demonstradas no quadro 2, as associaçóes dos descritores e seus respectivos quantitativos por base de dados.

Quadro 2: Associação de descritores com seus respectivos quantitativos por bases de dados

\begin{tabular}{|c|c|c|c|}
\hline \multirow{2}{*}{$\begin{array}{l}\text { Associaçáo de } \\
\text { descritores }\end{array}$} & \multicolumn{3}{|c|}{ Bases de Dados } \\
\hline & LILACS & BDENF & SCIELO \\
\hline $\begin{array}{l}\text { Sistemas de informação } \\
\text { AND Pré-natal }\end{array}$ & 22 & 03 & 25 \\
\hline $\begin{array}{l}\text { Pré-natal AND } \\
\text { Assistência à saúde }\end{array}$ & 25 & 01 & 250 \\
\hline Pré-natal AND & & & \\
\hline $\begin{array}{l}\text { Humanização da } \\
\text { assistência }\end{array}$ & 07 & 06 & 32 \\
\hline $\begin{array}{l}\text { Sistemas de informação } \\
\text { AND Assistência à } \\
\text { saúde }\end{array}$ & 45 & 01 & 37 \\
\hline TOTAL & 99 & 11 & 344 \\
\hline
\end{tabular}

Depois da leitura dos resumos das publicaçóes encontradas pela associaçáo dos descritores, foram selecionados oito artigos para leitura e análise, a fim de obter resposta pertinente à questâo de pesquisa. Desses artigos eleitos, seis foram encontrados na LILACS e dois no SciELO. Os artigos encontrados na BDENF não se enquadraram no perfil de obras procuradas para construção da pesquisa.

\section{Resultados e discussão}

\section{A assistência pré-natal}

A assistência pré-natal tem como finalidade a redução da morbidade e mortalidade materna e infantil ${ }^{7}$ por meio da implementação de medidas e realização de procedimentos mínimos definidos pelo PHPN. O programa estabelece os seguintes procedimentos a serem realizados no acompanhamento da gestação: início da assistência pré-natal até o quarto mês de gestação (16 semanas); mínimo de seis consultas para gestantes 
sem complicaçôes ${ }^{8}$, uma no primeiro trimestre, duas no segundo e três no terceiro; rotina de exames - sendo solicitados na primeira consulta: tipagem sanguínea, dosagem de hemoglobina $(\mathrm{Hg}) /$ hematócrito $(\mathrm{Ht})$, glicemia, VDRL, anti-HIV e exame de urina (EAS), e no início do terceiro trimestre gestacional: VDRL, glicemia e EAS; vacinação antitetânica; consulta puerperal ${ }^{9}$.

Os resultados do período perinatal estão relacionados a uma série de fatores que inclui determinantes biológicos, socioeconômicos e de origem assistencial? A assistência pré-natal contribui para resultados favoráveis, pois tem como algumas finalidades a detecção e o tratamento de afecçóes, além de controle dos fatores de risco que podem trazer complicação para a saúde da gestante e do feto9. Para que a assistência pré-natal seja efetiva na manutenção de uma gestação de baixo risco, prevenção e recuperação da saúde, é necessário que os profissionais da saúde saibam o que é preconizado pelo PHPN.

A avaliação da cobertura e adequação das consultas de pré-natal é uma ferramenta imprescindível e norteadora das açóes de gestores e profissionais da saúde ${ }^{10}$. Por meio da constante avaliação dos sistemas de saúde, será possível a reorganizaçáo e o planejamento dos serviços, visando à melhoria da assistência, manutenção da qualidade e humanização dos atendimentos. Uma assistência humanizada no pré-natal é o primeiro passo para desfechos favoráveis, a começar pelo nascimento saudável. Um cuidado humanizado deve objetivar fundamentalmente minimizar a incidência de mortes materna e infantil, além de preparar para a maternidade e paternidade, fornecer autonomia e proporcionar vivência segura do processo de nascimento ${ }^{11}$.

De acordo com alguns estudos realizados, percebe-se uma falha na cobertura da assistência pré-natal. Mesmo que a grande maioria das gestantes receba esse atendimento, há ainda a dificuldade da aderência de alguns procedimentos fundamentais, principalmente no que diz respeito ao início precoce e número de consultas. Muitas gestantes começam o acompanhamento pré-natal tardiamente e, consequentemente, a quantidade de consultas é reduzida até o fim da gestação. Outro determinante que deve ser considerado é a qualidade da assistência pré-natal. As análises embasadas no quantitativo de consultas somente podem ocultar graves problemas na qualidade do cuidado ofertado?.

O cuidado pré-natal adequado oferecido nos serviços de saúde está relacionado à qualidade da assistência prestada pelos profissionais, visando o seguimento do que é preconizado no Programa de Humanização no Pré-Natal e Nascimento, além de estar vinculado à análise constante do atendimento oferecido. Os sistemas de informação em saúde (SIS) constituem ferramentas essenciais para a manutenção da assistência de qualidade.
Os SIS utilizados para obtenção de informações durante o pré-natal é o SISPRENATAL.

\section{O sistema pré-natal (SISPRENATAL)}

O PHPN quando foi criado, além de definir os procedimentos mínimos para assistência pré-natal de qualidade, estipulou em primeiro momento o repasse de incentivo financeiro, transferidos do Fundo Nacional de Saúde, para os municípios a cada gestante que cumprisse e passasse por essas intervençóes do programa ${ }^{12}$.

O SISPRENATAL, por sua vez, servindo como instrumento de gestão, permite, por meio da monitoração do cumprimento das açôes mínimas essenciais para assistência pré-natal adequada, que haja a "implementação de repasse de verbas aos municípios que cumpram esses requisitos mínimos" ${ }^{12}$. Esta seria uma estratégia para incrementar o cumprimento das metas estabelecidas por meio do estímulo financeiro.

Por meio do SISPRENATAL, o Ministério da Saúde do Brasil monitora o cumprimento das açôes mínimas contempladas no programa e repassa verbas para municípios que cumprem esses requisitos. A disponibilização de recurso financeiro para investimento regional é fundamental para a organização do cuidado e para a qualidade do serviço de saúde ${ }^{13}$.

Hoje em dia, mais do que o monitoramento nacional da atenção à gestante e instrumento para o repasse financeiro a essa assistência, o SISPRENATAL tem como finalidade a obtenção de informação em saúde durante o pré-natal, parto e puerpério. Esta ação é fundamental para a avaliaçáo dos cuidados prestados em diferentes contextos. Se forem precisas, as informaçóes obtidas podem refletir o panorama diverso da saúde materna no Brasil, permitindo investimentos em nível local, regional e universal, com especificaçóes para os diferentes grupos populacionais e contextos sociais ${ }^{12}$.

$\mathrm{O}$ registro da inserção das gestantes no PHPN, por meio do SISPRENATAL, utiliza dois documentos como entrada de dados, a Ficha de Cadastramento da Gestante e a Ficha de Registro Diário dos Atendimentos das Gestantes, que podem ser efetuados na unidade de atendimento, no distrito sanitário, na Secretaria Municipal de Saúde ou na Secretaria Estadual de Saúde, em nível regional ou central, permitindo a importação dos dados registrados em todas as esferas, possibilitando a sua concretização final pelo SUS ${ }^{14}$. A alimentação desse sistema é de responsabilidade dos gestores dos municípios. É relevante que os profissionais sejam treinados para o efetivo preenchimento do banco de dados e minimização de possíveis falhas. 
A melhoria do cadastramento das gestantes ao PNPH depende de uma organização gerencial que possibilite o treinamento e a conscientização dos profissionais responsáveis por desenvolverem esta função. Os problemas de cadastramento das gestantes no SISPRENATAL podem estar relacionados com o encaminhamento das fichas destinadas ao cadastramento no Programa ${ }^{14}$.

De acordo com alguns estudos realizados, apesar de ser uma ferramenta que norteia a ação de gestores e profissionais da saúde, o cadastramento das gestantes no SISPRENATAL ainda é falho e representa um desafio para o PHPN ${ }^{14}$. É possível afirmar que, em alguns serviços, há deficiência na qualidade das informaçôes geradas e esses erros podem ser provenientes do desconhecimento do profissional que manipula o sistema ainda pelo caminho percorrido pela informação. O sistema é alimentado a partir de planilhas preenchidas pela unidade de saúde, após cada atendimento. Desta forma, podem ser geradas falhas de documentação, seja no detalhamento da planilha, no envio ou na posterior digitação no sistema. "Em geral, o funcionário da unidade responsável pelo registro das informaçóes nas planilhas do SISPRENATAL não participa da consulta que gerou tais dados"13.

Ainda que haja falhas no sistema pré-natal, é notório que este traga benefícios para a assistência à saúde da gestante, visto que tem como finalidades obter informações sobre a saúde da mulher durante gestação, parto e puerpério. É essencial para o financiamento dessa assistência, pois possibilita o repasse de verbas aos fundos municipais para cada gestante que cumpra as açóes mínimas preconizadas pelo Programa de Humanização no Pré-Natal e Nascimento, que são o início do acompanhamento com até 120 dias de gestação, realização de seis consultas pré-natais e uma consulta puerperal, recebimento da vacina antitetânica e realizaçôes de exames laboratoriais.

Faz-se necessário, portanto o correto preenchimento de planilhas que alimentem o sistema, visando à redução de possíveis subnotificaçóes. Os gestores municipais são os responsáveis pelo funcionamento desse sistema. É imprescindível que os profissionais que alimentam o sistema sejam devidamente treinados para o sucesso do preenchimento do banco de dados, assim como a avaliação constante do cadastramento das gestantes. A avaliação do funcionamento, além de contribuir com apontamento de acertos e/ou falhas, possibilita o delineamento de soluçóes, reorganização de atividades e serviços, apontando caminhos alternativos, com o intuito de maximizar a utilização de recursos disponíveis ${ }^{14}$.

A partir do contexto trazido pela pesquisa, pode-se afirmar que este sistema é uma ferramenta facilitadora da assistência pré-natal, pois possibilita a obtenção de informaçōes sobre a saúde da mulher no período gestacional. A partir de sua implementação e utilização correta pelos sistemas de saúde, torna-se possível a organizaçáo e o planejamento de estratégias que visem maior aderência às determinaçóes do PHPN e, mediante a adequação dessas determinaçóes, a toda ou maioria das gestantes. Os desfechos positivos do período perinatal serão elevados assim como a qualidade da assistência prestada.

\section{Conclusão}

Por meio da revisão integrativa realizada neste estudo, pode-se constatar a significativa quantidade de publicações encontradas nas bases científicas de dados que abordam o tema cuidado de gestante e SISPRENATAL, além de se observar as falhas encontradas na cobertura da assistência pré-natal. Por mais que grande parte das gestantes realize o pré-natal, procedimentos como o início precoce das consultas - que deveria acontecer até no máximo 120 dias do início gestação - e um número mínimo de seis consultas ainda são prejudicados devido à falta de aderência dessas clientes, que faz com que o seu cadastramento fique prejudicado no sistema, devido ao início tardio ou número de consultas mínimas não realizadas.

Deve-se ter ciência de que os sistemas de informação em saúde são ferramentas de grande importância para a manutenção de uma assistência de qualidade. Neste caso, o sistema é o SISPRENATAL, que é um instrumento de gestão utilizado para incrementar o cumprimento das metas estabelecidas por meio de estímulos financeiros.

O SISPRENATAL possui como entrada de dados a Ficha de Cadastramento da Gestante e a Ficha de Registro Diário dos Atendimentos delas. É relevante que os profissionais de saúde, que lidam com esse tipo de assistência, sejam treinados para que possam realizar o correto preenchimento das fichas. Visto que foi observado durante a elaboração da pesquisa que o cadastramento das gestantes ao SISPRENATAL apresenta-se falho, podendo ocorrer devido ao desconhecimento dos profissionais que lidam com essa assistência. Embora haja falhas, é importante frisar que o sistema, quando utilizado adequadamente pelo serviço de saúde, traz benefícios à saúde da gestante, pois possui como finalidades obter informaçôes sobre a saúde da mulher durante a gestação, parto e puerpério. Para que ocorra a manutenção do acompanhamento, é necessário que haja o financiamento da assistência, que é realizado pelo repasse de verbas aos municípios a cada gestante que cumpre as ações preconizadas, que são o início do pré-natal em até 120 dias de início de gestação, realização mínima de 
seis consultas de pré-natal, uma consulta puerperal, realização da vacina antitetânica e de exames laboratoriais.

O SISPRENATAL possibilita, por meio das informaçóes geradas, que os gestores em saúde sejam capazes de planejar e (re)organizar os serviços de saúde voltados à saúde materno-infantil, tendo como finalidade primordial a melhoria e a manutenção da assistência em seu mais alto nível de qualidade.

Agradecimentos: Agradecemos à disciplina de Gerenciamento dos Sistemas de Informação em Saúde por proporcionar ao aluno um conhecimento mais aprofundado sobre os sistemas utilizados no Sistema Unico de Saúde e ao curso de Enfermagem da Escola de Enfermagem Aurora de Afonso Costa por oferecer aos alunos vivências no cotidiano da profissão ainda como acadêmicos de Enfermagem, o que gera, como consequência, questionamentos em relação à prática profissional e o surgimento de pesquisadores na área de Enfermagem.

\section{Referências}

1. Brasil. Ministério da Saúde. Guia de Vigilância epidemiológica. 6 ed. Brasília - DF; 2005.

2. Pinheiro ALS. Gerência de Enfermagem em Unidades Básicas: a informação como instrumento para a tomada de decisão. Rev APS. 2009; 12(3): 262-70.

3. Brasil. Ministério da Saúde. Implantação do Programa de Humanização no Pré-natal e Nascimento. Brasília - DF; 2012.

4. Brasil. Ministério da Saúde. O SISPRENATAL. Brasília DF; 2012.

5. Minayo MCS. O desafio do conhecimento pesquisa qualitativa em saúde. 10 ed. São Paulo: Hucitec; 2007.

6. Mendes KDS, Silveira RCCP, Galvão CMG. Revisão Integrativa: método de pesquisa para a incorporação de evidências na Saúde e na Enfermagem. Texto Contexto Enferm, Florianópolis. 2008; 17(4): 758-64.

7. Silva EP, Lima RT, Ferreira NLS, Costa MJC. Pré-natal na atenção primária do município de João Pessoa-PB: caracterização de serviços e usuárias. Rev Bras Saúde Matern Infant, Recife. 2003; 13(1): 29-37.

8. Coutinho T, Monteiro MFG, Sayd JD, Teixeira MTB, Coutinho CM, Coutinho LM. Monitoramento do processo de assistência pré-natal entre as usuárias do Sistema Único de Saúde em município do Sudeste brasileiro. Rev Bras Ginecol Obstet. 2010; 32(11): 563-9.

9. Domingues RMSM, Hartz ZMA, Dias MAB, Leal MC. Avaliação da adequação da assistência pré-natal na rede SUS do município do Rio de Janeiro, Brasil. Cad Saúde Pública, Rio de Janeiro.2012; 28(3):425-37

10. Anversa ETR, Bastos GAN, Nunes LN, Dal Pizzol TS. Qualidade do processo da assistência pré-natal: unidades básicas de saúde e unidades de estratégia saúde da família em município no Sul do Brasil. Cad Saúde Pública, Rio de Janeiro. 2012; 28(4): 789-800.

11. Zampieri MFM; Erdmann AL. Cuidado humanizado no pré-natal: um olhar para além das divergências e convergências. Rev Bras. Saúde Matern. Infant, Recife. 2010; 10(3): 359-67.

12. Andreucci CB, Cecatti JG. Desempenho de indicadores de processo do Programa de Humanização do Pré-Natal e Nascimento no Brasil: uma revisão sistemática. Cad Saúde Pública, Rio de Janeiro. 2011; 27(6): 1053-64.

13. Andreucci CB, Cecatti JG, Macchetti CE, Sousa MH. Sisprenatal como instrumento de avaliação da qualidade da assistência à gestante. Rev Saúde Pública. 2011; 45(5): 854-63.

14. Moimaz SAS, Garbin CAS, Garbin AJI, Zina LG, Yarid SD, Francisco KMS. Sistema de Informação Pré-Natal: análise crítica de registros em um município paulista. Rev Bras Enferm. 2010 Mai-Jun; 63(3): 385-90. 